\title{
Die Küste \\ als transkultureller Erinnerungsraum in W.G. Sebalds Die Ringe des Saturn
}

DORIT MÜLLER

\begin{abstract}
In W.G. Sebald's The Rings of Saturn a walk along the coast of East Anglia triggers numerous memories about catastrophic events, leading to a variety of stories about people living under conditions of transit, exile, homelessness, and precarious identity. It is no coincidence that all these people are connected with the coast, a paradigmatic motif for processes of erosion and extinction, exchange and amalgamation, border-crossing and hybridity. This article investigates the narrative construction of the coast as a place for transcultural memory and describes the different functions of this specific literary scene for structuring and making visible processes of identity negotiation in Sebald's text. Furthermore, the article focuses on the coast's potential to provoke reflection upon processes of imagination and multiperspectival writing.
\end{abstract}

Title: The Coast as a Place of Transcultural Memory in W.G. Sebald's The Rings of Saturn

Keywords: Sebald, W.G. (1944-2001); memory; coast; liminal zone; identity

\section{EINFÜHRUNG}

W.G. Sebalds 1995 erschienener Prosatext Die Ringe des Saturn, welcher sich im Untertitel als Eine englische Wallfahrt ausgibt, stellt eine Ansammlung von Reiseeindrücken dar, die ein melancholischer Ich-Erzähler auf einer Wanderung durch die ostenglische Grafschaft Suffolk macht. In zehn Kapiteln werden Schauplätze der durchreisten Landschaft vorgestellt - heruntergekommene Provinzstädte, verfallene Landsitze, marode Befestigungstürme am Strand, ein unbenutzter reading room für Seefahrer, im Meer verschwundene Landschaften, Küstenorte, die ihre Blüte im Mittelalter hatten. Neben dem Adelssitz Somerleyten besucht der Erzähler die Ortschaften Lowestoft, Dunwich, Southwold, Middleton, Woodbridge und Orford. Sie sind der Ausgangspunkt weit ausgreifender und zugleich eng verflochtener Erinnerungen und Erzählungen über das Leben und Sterben historischer und fiktiver Persönlichkeiten, die auf eine bestimmte Weise mit dem spärlich besiedelten Küstenstrich von Suffolk verbunden sind und deren kulturelle Vernetzungen von England über Polen 
und Russland bis nach China und in den Kongo, nach Belgien, den Niederlanden, Frankreich und Deutschland reichen. Es sind vor allem Binnenerzählungen über Grenzgänger*innen, deren kulturelle Identität seit jeher in Zweifel gezogen wurde oder problematisch geworden ist, die sich der eigenen Kultur entfremdet haben oder deren Assimilation in einer neuen Umgebung nicht oder nur schwer gelingt. Sebalds Text kann als Versuch verstanden werden, die Grenzgänge dieser Figuren zwischen den Kulturen zu rekonstruieren und die Graduationen der jeweiligen kulturellen Verhältnisse auszuloten. Zugleich bietet er Lesarten für die individuellen Geschichten an, indem er sie in das übergreifende Narrativ einer kollektiven Geschichte der Zerstörung und Auslöschung, aber auch einer »Fähigkeit zur Transmigration« (Sebald 2015: 39) im Sinne einer ständigen Transformation von Daseinsweisen einbettet.

Dass die Erinnerungen und Erzählungen auf einer Wanderung an der ostenglischen Nordseeküste entstehen, scheint kein Zufall zu sein. Denn die Küste steht paradigmatisch für Prozesse der Erosion und Auslöschung, für Austausch und Vermischungen, für Grenzgänge und Hybridisierungen. Nur im Medium der Kartographie erscheint die Küste als eine Grenzlinie, die Festland und Meer säuberlich trennt. Im Grunde genommen erweist sie sich jedoch als ein Zwischenraum »mit überaus unscharfen Rändern [...], an denen sich die Elemente auf verschiedene Weise durchdringen« (Feldbusch 2003: 12). Meer und Land bekämpfen und versöhnen sich hier beständig. Die Küste ist »dem Lande und dem Meer gegenüber nur ein Übergang, eine Vermittlung, sie steht nicht für sich« (Ratzel 1911: 34). Das rasch wechselnde »Pendeln der jeweiligen Uferlinien« (Kelletat 1999: 84), die Strömungsverhältnisse, Überschwemmungen, Hebungen und Senkungen des Meeresspiegels sorgen für zerfranste und zerklüftete Küstensäume, so dass selbst Küstenmorphologen auf starre Definitionen der Grenze verzichten. Auch maritime Grenzbereiche wie Flussmündungen, Felsvorsprünge, Vorgebirge oder künstliche Befestigungsanlagen erfahren beständig Veränderungen, da sie Wind, Wetter und Meeresströmungen unterworfen sind.

Sebalds Prosatext thematisiert eben diesen Prozess. Wiederholt ist von den Erosionen der Küstenregion die Rede, welche durch die »Gewalt des unablässig die Küste zerfressenden Meers« hervorgebracht sei und dazu führe, »daß monatelang kein Mensch mehr wußte, wo die Grenze war zwischen dem Meer und dem Land « (Sebald 2015: 189f.). In einer traumartigen Sequenz nimmt der IchErzähler wahr, wie »von der Küste nach Süden hinunter ganze Landesteile abgebrochen und in den Wellen versunken waren « (ebd.: 207). Sein Blick liegt auf dem »stets von Einbrüchen bedrohten Rand des festen Landes« (ebd.: 83) oder schweift über »im Dunst der Ferne« (ebd.: 274) verschwimmende Ufer.

Die Beschreibungssprache verweist nicht nur auf die Fragilität und Dynamik des Küstenraumes, sondern auch auf die Unschärfe seiner Ränder. Wo das Meer aufhört und das Festland anfängt, scheint eine Frage der Perspektiven, Motivationen und Interessen jener zu sein, die das >Dazwischen < beobachten und vermessen, besiedeln oder durchschreiten, mit Mythen besetzen und kulturell codieren - die Seeleute und Meereskundler*innen, Küstenbewohner*innen, Reisenden und Schriftsteller*innen. Wie die Forschung herausgestellt hat, sind 
vor allem Letztere prädestiniert dafür, den maritimen Grenzbereich als »Erlebnisort, Erzählraum und Bild für Erfahrungen und Projekte« (Feldbusch 2003: 15) zu thematisieren. In ihren Umschreibungen manifestiert sich die Denkfigur eines Grenzraums, der die Logik des Entweder-oder überwindet und einen Zwischenraum beschreibt, in dem es »zu einer Art Überblendung der angrenzenden Gebiete kommt « (Wirth 2012: 19). Man kann ebenso von einer Schwelle zwischen zwei Welten sprechen oder die Küste als »wandelbare Zone intensiven Austauschs « im Sinne eines natürlichen und kulturellen »Grenzregime[s] von Erosion, Produktion und Kommunikation« (Wolf 2013: 337) verstehen.

Sebalds Ich-Erzähler scheint dieses Konzept aufzugreifen. Während er den erodierenden Küstenraum wandernd erkundet, kommen ihm Reminiszenzen, Anekdoten, Traumbilder und Geschichten in den Sinn, die einerseits eine kaleidoskopische Ansicht der Katastrophen- und Leidensgeschichte der Menschheit entstehen lassen, andererseits aber auch die produktiven Momente und kulturellen Verflechtungen als Kehrseite der Erosion und Auflösung sichtbar machen. So versetzt ihn der Blick auf das Meer in das Kampfgeschehen großer Seeschlachten des 17. Jahrhunderts (vgl. Sebald 2015: 94) und zugleich in die Betrachtung berühmter Seeschlachtengemälde (vgl. ebd.: 95). Er führt ihn zu giftigen Algenfeldern und ausgefischten Meeren (vgl. ebd.: 69), gaukelt ihm aber auch phantastische Welten mit Seeungeheuern vor (vgl. ebd.: 89). Die Strandwanderung erinnert ihn an Überschwemmungskatastrophen und Migrationsbewegungen, aber auch an die Küste als »Wallfahrtsort für schwermütige Schriftsteller« (ebd.: 192). Nicht selten avanciert sie zum Fluchtort für (fast ausschließlich männliche) Aussteiger und Exzentriker. Es überrascht nicht, dass die an den Küstenorten aufkommenden Erinnerungen und Assoziationen an Geschichten von Personen gebunden sind, die an der Schwelle zu fremden Kulturen stehen, weil sie Verfolgte und Vertriebene sind oder sich der eigenen Kultur entfremdet haben. Der Erzähler, der deutliche Züge seines Autors trägt, gehört selbst zu jenen transitorischen Gestalten, die zwischen den Kulturen ihrer Herkunft und ihres Aufenthaltes vermitteln müssen. Aus dieser Lebenssituation speist sich offensichtlich auch seine Empathie für jene, die neue kulturelle Identitäten annehmen mussten, weil sie wie Michael Hamburger aus dem nationalsozialistischen Deutschland ins Exil getrieben wurden oder wie der polnische Patriot Apollo Korzeniowski jahrelang in der russischen Verbannung ausharren mussten. Das Interesse gilt gleichermaßen den ruhelosen, exzentrischen und oft traumatischen Lebenswegen von gestrandeten Intellektuellen, Dichtern oder Staatsbeamten, die kulturelle Randexistenzen führten, weil sie ihre Herkunft verleugneten, alternative Lebensformen erprobten oder den Völkermord anklagten, wie der irische Diplomat Roger Casement.

Die Küste als maritimer Grenzraum scheint in Sebalds Text demnach mehrere Funktionen zu übernehmen: Als konkreter Schauplatz und Erlebnisraum einer literarischen Erzählerfigur strukturiert sie sowohl deren Bewegung durch den geographischen Raum als auch die Abfolge ihrer Eindrücke und Gedanken. Des Weiteren fungiert die Küste als Erinnerungsanlass und Gedächtnisort. Dass sich dabei die Grenzlinien permanent verändern, auflösen und neu bil- 
den, ist eine Spezifik, die der assoziativen Verknüpfung des Erinnerten, seinem Verschwinden und unerwarteten Wiederauftauchen korrespondiert. Schließlich stellt die Küste einen Aushandlungsort transkultureller Prozesse dar. Gemeint sind damit Formen der »Fluidität«, der »Dynamik « und »Grenzüberschreitungen zwischen den Kulturen « (Kimmich/Schahadat 2012: 7), wie sie nicht erst im Zeitalter der Globalisierung signifikant geworden sind.

In der Bewegung durch die »liminale Zone « ${ }^{1}$ zwischen Land und Meer führt der Ich-Erzähler vor, wie der Blick auf die See Narrationen freisetzt, die verschiedene kulturelle Räume und deren Bewohner*innen in Beziehung setzen, deren kulturelle Transformationen und Verflechtungen aufzeigen, aber auch den Prozess des Geschichtenerzählens selbst zum Gegenstand der Reflexion machen. Zumindest gibt die Sicht von der Klippe auf das Meer dem Ich-Erzähler Anlass, seine Gedanken um die Möglichkeiten und Grenzen des Imaginierens von Welten kreisen zu lassen und Jorge Louis Borges' »labyrinthische Konstruktion Tlöns« (Sebald 2015: 91) in Erinnerung zu rufen.

In der umfänglichen Forschungsliteratur wurden Sebalds Ringe des Saturn vor allem als Ausdruck eines melancholischen Erinnerns (vgl. Lemke 2008) und katastrophischen Schreibens (vgl. Mosbach 2008) gedeutet, in dem sich die »zivilisatorischen Abirrungen « (Öhlschläger 2006: 312) und eine »Absage an die Fortschrittsräume der Moderne« (Albes 2002: 280) offenbaren. Zwar hat man durchaus die Bezüge zwischen Wanderung und Schreibakt untersucht (vgl. Öhlschläger 2017: 4of.), doch darauf verzichtet, sich den Zusammenhang zwischen Küstentopos und literarischer Erinnerungsarbeit anzuschauen. Auch ist die Küste bisher nicht in ihrer Funktion als Erzählraum transkultureller Erinnerungen in den Blick geraten. ${ }^{2}$ Der vorliegende Beitrag wird sich deshalb der beschriebenen Küstenpoetik in Sebalds Erzählung in mehreren Schritten nähern. Zunächst analysiert er die Spezifik der aus der Küstenwanderung des Erzählers hervorgehenden Erinnerungsarbeit, an der Text und Bild gleichermaßen beteiligt sind. Zweitens fragt er nach den narrativen Verfahren, mit denen der Küstenraum als Gedächtnisort transkultureller Prozesse konstruiert wird. Nicht zuletzt lenkt er den Blick auf den Küstenraum als Reflexionsraum imaginativer Prozesse und multiperspektivischen Schreibens.

\section{Der Küstenraum als Ausgangspunkt LITERARISCHER ERINNERUNGSARBEIT}

Von der »Fußreise« entlang der ostenglischen Küste verspricht sich der Erzähler, »nach dem Abschluß einer größeren Arbeit« der in ihm sich »ausbreitenden

1 | Vgl. zum Konzept der "liminalen Zone" (Bonner/Egger/Hess-Lüttich 2016: 13) bzw. des "Transitraumes" als "Vorstellung des Übergangs, des Transitorischen, der Transformation, des Hybriden " (ebd.: 11) die Einführung zum Schwerpunktthema Transiträume der Zeitschrift für interkulturelle Germanistik.

2 | Vgl. den Forschungsüberblick bei Feldbusch (2003: 16-57). 
Leere entkommen zu können« (Sebald 2015: 11). Der erhoffte Blick auf das Meer mag dabei Antrieb gewesen sein. Gilt doch die Strandwanderung seit Gründung von Seebädern im 18. Jahrhundert als »Heilmittel gegen die Melancholie und Nervosität«, die Küste als »privilegierter Ort der Selbstfindung« (Bader 2008: 23f.). ${ }^{3}$ An der Nahtstelle zwischen Land und Meer kann sich laut medizinischer Gutachten der städtische Körper dem Rhythmus des Meeres überlassen und seine Empfindsamkeit erproben (vgl. Corbin 1999: 84-97). Sebalds Ich-Erzähler scheint jedoch gegen solche Therapievorschläge immun zu sein. Zwar erfüllt sich die Hoffnung auf Überwindung der Leere »bis zu einem gewissen Grad« (Sebald 2015: 11), denn selten habe sich der Erzähler »so ungebunden gefühlt wie damals bei dem stunden- und tagelangen Dahinwandern durch die teilweise nur spärlich besiedelten Landstriche hinter dem Ufer des Meers« (ebd.). Andererseits ist von einem »lähmenden Grauen« die Rede, das den Erzähler »verschiedentlich überfallen hatte angesichts der selbst in dieser entlegenen Gegend bis weit in die Vergangenheit zurückgehenden Spuren der Zerstörung« (ebd.). Die Wanderung steht »unter dem Zeichen des Hundssterns« (ebd.), das der Erzähler rückblickend als Auslöser einer melancholischen Grundstimmung deutet.

Die Wanderung perpetuiert zunächst eben jenen Mangel, den sie beheben soll. Der vor der inneren Leere flüchtende Erzähler begegnet unterwegs dem äußeren Abbild seiner inneren Verfassung: »Nichts ist hier zu sehen, als ab und zu ein einsames Flurwächterhaus, als Gras und wogendes Schilf« (ebd.: 42); leer sind die Bahnhöfe und die Perrons, die verwahrlosten Städte und die heruntergekommenen Hotels; »nirgends ein Mensch, den man nach dem Weg hätte fragen können « (ebd.: 43). Die Erfahrung der Leere und des Stillstands kulminiert schließlich im Anblick des Meeresstrands, der sich »irgendwo zwischen dunkel und hell« erstreckt: »[E]s bewegte sich nichts, weder in der Luft, noch auf dem Wasser. Selbst die schneeweiß in der Bucht auflaufenden Wellen, so schien es mir, standen still.« (Ebd.: 59)

Die der Textpassage beigefügte Fotografie - wie übrigens fast alle der fotografischen Abbildungen der Küste im Buch - scheint diese Beobachtung nicht nur zu illustrieren, sondern aufgrund ihrer Unschärfe und Beliebigkeit hinsichtlich des Aufnahmeortes als Allegorie der Vergänglichkeit und Auslöschung zu fungieren (Abb. 1). Qualifiziert als Ausschussprodukte, die Zerfall auch mittels der eigenen verschwindenden Materialität ausstellen, erweisen sich die Aufnahmen des Meeres als »Mementos einer im Zerstörungsprozeß und im Verschwinden begriffenen Welt« (Sebald 1985: 178).

Nun ist es aber paradoxerweise gerade die sich im Bild des Meeres und seiner Umgebung manifestierende Leere, welche eine ausgeprägte Erinnerungs- und Einbildungskraft des Erzählers antreibt. Ausgehend von der trostlosen Verlassenheit und ruinösen Beschaffenheit der Küstengebiete erläutert er kulturelle, wirtschaftliche und historische Zusammenhänge, ruft philosophische und literarische Traditionen auf, setzt weit auseinanderliegende Ereignisse und Personen in ein Verhältnis. Diese stehen in einem mehr oder weniger engen Bezug

3 | Siehe dazu auch den Artikel von Michael Niehaus in diesem Heft. 
zur Küstenlandschaft, zu ihren tatsächlichen oder phantasierten Eigenschaften und zu den Bildern, die die Wahrnehmung des Meeres prägen. Die vermeintlich leere Landschaft scheint so reich an Zeichen, dass sie für den Erzähler zu einem Text der Vergangenheit wird, den er mittels Erinnerungen lesbar macht. Meist ist es der Meerblick, der den Erinnerungsprozess in Gang setzt. Im vierten Kapitel glaubt der Erzähler beim abendlichen Strandspaziergang in Southwold plötzlich vor einer Theaterbühne zu stehen:

Ich fühlte mich wie in einem leeren Theater, und es hätte mich nicht gewundert, wenn vor mir auf einmal der Vorhang aufgegangen und auf dem Proszenium beispielsweise der 28. Mai 1672 wieder heraufgekommen wäre, jener denkwürdige Tag, an dem dort draußen die holländische Flotte, das strahlende Morgenlicht hinter sich, aus dem über der See treibenden Dunst auftauchte und das Feuer eröffnete auf die in der Bucht vor Southwold versammelten englischen Schiffe. (Sebald 2015: 94)

Abbildung 1: Küste als Allegorie der Leere (Sebald 2015: 59)

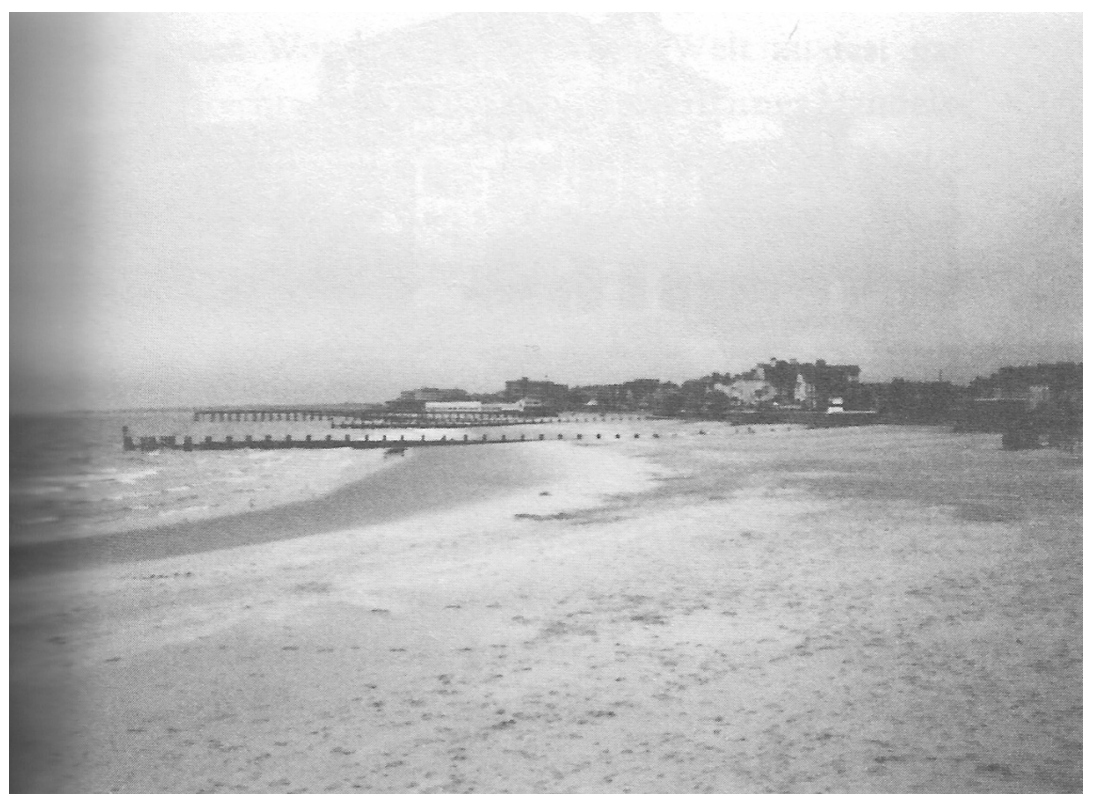

Der inszenierte Schauplatzwechsel von der Küste ins Theater läutet zugleich einen Zeitenwechsel ein: Versetzt vom 20. ins 17. Jahrhundert fragt der Erzähler sich, wie wohl die damaligen Küstenbewohner »das seltene Schauspiel vom Strand aus verfolgt« (ebd.) haben könnten, und kommt dann auf die grundsätzliche Unzuverlässigkeit von Geschichtsüberlieferungen zu sprechen. Das Thema, das für alle Texte Sebalds substantiell ist, wird hier am Beispiel von Seeschlachtengemälden exemplifiziert, die trotz aller realistischen Absichten immer »pure Fiktionen « bleiben müssten, da sie die »erlittene Pein « und das »gesamte Werk der Zerstörung« (ebd.) durch die Schlachten nicht annäherungsweise ins Bild 
setzen könnten. Aus dieser kulturkritischen Betrachtung, an die sich lakonische Mutmaßungen über die kontingenten Ursachen maritimer Vorherrschaft knüpfen, kehrt der Ich-Erzähler zur unmittelbaren sinnlichen Wahrnehmung zurück. So erblickt er über dem Meer »eine seltsam geformte Wolkenbank«, deren »wie die Eisfelder des Kaukasus « gleißende »Gipfelregionen « wiederum einen Traum ins Gedächtnis rufen, in dem er »ein ebensolches fernes und fremdes Gebirge seiner ganzen Länge nach durchwandert hatte«, durch »Schluchten, Tobel und Täler, über Jochstraßen, Halden und Driften« (ebd.: 98).

Die Passagen zeigen auf exemplarische Weise, wie aus Beobachtungen, Reflexionen, Imaginationen und traumartigen Sequenzen, die sich aus dem maritimen Grenzraum speisen, ein Erinnerungszusammenhang gestiftet wird, der auseinanderliegende Räume, Zeiten, Wahrnehmungszustände und Themen zusammenführt. Neben Erzähltechniken der Analepse und Prolepse, des Schauplatz- und Medienwechsels spielen vor allem die Verfahren der Variation und Wiederholung eine zentrale Rolle. Wird die sinnliche Erfahrung eines Meerwolkengebildes durch die Erinnerung an die Durchquerung einer geträumten Landschaft gespiegelt, so wiederholt sich die gesamte Küstenwanderung durch häufig aufgerufene Erinnerungen an zurückliegende Reisen des Erzählers und seiner Figuren im Sinne einer Mise en abyme-Struktur. Im fünften Kapitel beispielsweise, das die Kolonialisierungsgeschichte des Kongo beschreibt, erinnert sich der Erzähler an eine Belgienreise in den 196oer Jahren, welche ihn nach Brüssel führte, dessen Stadtbild die zuvor beschriebenen Verbrechen der Kolonisatoren des Kongo wiedererkennen ließ. Die Strandwanderung auf dem Gunhill von Southwold wird wiederum mit einer Reise kurzgeschlossen, die Sebald genau ein Jahr zuvor von Basel nach Amsterdam und Den Haag unternommen hatte. Sie führte ihn unter anderem zu Rembrandts berühmtem Gemälde Die Anatomie des Dr. Tulp, das wiederholt im Text aufgegriffen und als Symbol eines menschenfeindlichen Forschungsdrangs gedeutet wird. Im siebten Kapitel schließlich lässt ihn die Betrachtung der verödeten Heidelandschaft von Dunwich, deren traurigen Zustand er auf die anthropogene Zerstörung dichter Wälder zurückführt, an eine Griechenlandreise erinnern, bei der er Zeuge eines Flächenbrandes wurde.

Solche »Mechanismen der Wiederholung (Albes 2002: 280) bedingen kulturräumliche Verschachtelungen und Überlagerungen unterschiedlicher Zeitkonzepte. Wenn der Erzähler im vierten Kapitel am Strand in Southwold einen früheren Blick vom holländischen Strand aus nach England erinnert, dann nimmt er damit nicht nur einen Perspektivwechsel vor, sondern vervielfältigt auch das gegenwärtige Erleben, indem er die Vergangenheit darüberlegt. Er erzeugt gewissermaßen eine »Gleichzeitigkeit des Ungleichzeitigen« (Öhlschläger 2017: 42). Zu dieser Verflüssigung zeitlicher Konzeptionen passt auch die grenzüberschreitende Vision des Ich-Erzählers am Strand von Scheveningen, die ihn in die biblische Geschichte von der Rettung der Israeliten zurückversetzt:

Ich hörte das Rauschen des Meers, verstand, halb im Traum, jedes holländische Wort und glaubte mich zum erstenmal in meinem Leben angekommen, zu Hause. Selbst im 
Erwachen schien es mir noch einen Augenblick, als halte rings um mich her mein Volk Rast auf dem Zug durch die Wüste. (Sebald 2015: 105)

Die Küste wird in diesem Erzählsetting zum kollektiven Erinnerungsort, weil sich hier mythische Erzählungen, kulturhistorische Bezugnahmen und symbolische Chiffren bündeln und transformieren können. Aufgrund seiner Intensität und sinnlichen Wirkung erzeugt das Meer Gefühle und Gedanken, welche die Form von Déjà-vu-Erlebnissen annehmen, andere Raum- und Medienassoziationen hervorrufen oder an kulturelle Erfahrungen in ähnlichen räumlichen Konstellationen erinnern. Die Spuren der Vergangenheit, die der Ich-Erzähler im Laufe seiner Reise entdeckt, sind dabei nicht nur textueller, sondern auch materieller Art. Sie verweisen auf eine kollektive Vergangenheit, die sich in der einst blühenden Küstenlandschaft abgelagert hat. So findet der Erzähler in den »verschandelten Fassaden und grotesken Vorgärtchen«, den »Spielsalons, Bingohallen, Betting Shops « der Küstenstadt Lowestoft die Zeugnisse eines jahrzehntelangen Niedergangs, der mit den »schweren Wirtschaftskrisen und Depressionen der dreißiger Jahre« (ebd.: 56 f.) seinen Anfang genommen habe. In Reedham sind es ein einsames Flurwächterhaus und Bruchstücke von Windmühlen, die sich »wie Mahnmale« (ebd.: 42) einer erloschenen Zivilisation ausnehmen. Die Ruinen der ins Meer reichenden militärischen Forschungsanstalt Orford schließlich scheinen ihm wie Überreste »unserer eigenen, in einer zukünftigen Katastrophe zugrundegegangenen Zivilisation« (ebd.: 282), über die ein Nachgeborener allen Grund haben würde, irritiert zu sein.

Das erinnernde Auffinden der Spuren, Reste und Bruchstücke beim Durchwandern des Küstenraumes, so wird spätestens hier deutlich, korrespondiert nicht nur den Prozessen der beständigen Zerstörung, Auslöschung und Transformation von Küstenlandschaften, sondern folgt auch einem spezifisch Sebald'schen Zugang zur Geschichte. In allen visuell und akustisch evozierten Erinnerungen, Traumsequenzen und materiellen Fundstücken zeigt sich die Menschheitsgeschichte als eine Geschichte vergangener, gegenwärtiger und zukünftiger Katastrophen. Man könnte mit Blick auf den Gesamttext geradezu von der Konstruktion eines »katastrophischen Mnemotops «(Pethes 2015: 200) ${ }^{4}$ sprechen, insofern der Text einen maritimen Erinnerungsraum entwirft, in dem alle Infrastrukturen und Architekturen, aber auch die Naturprozesse »als Wahrzeichen der destruktiven Energien der Zivilisationsgeschichte gelesen werden« (ebd.). Aus einer solchen Perspektive muss die Welt zum »absurden Raum« werden, »in dem die Fortschrittsideologie [nur] noch zufällige Restbestände des Schönen übrig gelassen hat« (Kastura 1996: 202). Diese zu sammeln und mit einer »Geste des Eingedenkens « (ebd.) $)^{5}$ dem kollektiven Gedächtnis zu überantworten, scheint das Konzept von Sebalds Schreibens zu sein.

4 | Pethes bezieht den Begriff des katastrophischen Mnemotops auf Sebalds Roman Austerlitz.

5 | Das Konzept des, Eingedenkensı übernimmt Sebald von Walter Benjamin: "Bekanntlich war es den Juden untersagt, der Zukunft nachzuforschen. Die Thora und das 
Welchen bewahrenswerten Beständen gilt aber nun die Sammelleidenschaft des Erzählers alias Sebald? Eine Antwort darauf findet sich im ersten Kapitel, das um die historische Person des frühneuzeitlichen Universalgelehrten Thomas Browne (1605-1682) kreist, der ein besonderes Interesse für singuläre Phänomene, Abnormitäten, abstruse Gestalten und Zwischenwesen entwickelte, vor allem aber die »Fähigkeit zur Transmigration « (Sebald 2015: 39) an Raupen und Faltern erforschte. Die Bezüge sind unverkennbar, denn auch auf Sebalds IchErzähler üben hybride Gestalten und außergewöhnliche Menschen, die sich in kulturellen Zwischenräumen aufhalten, eine besondere Faszination aus. Durch die erzählerische Annäherung an diese meist historisch vergessenen Exzentriker und kulturellen Grenzgänger etabliert der Text eine alternative Erinnerungskultur, die zum einen das Ausgegrenzte, Randständige und Gegenläufige, zum anderen die in diesen Figuren angelegte Verflechtung der Kulturen ins Zentrum der Gedächtnisarbeit rückt. ${ }^{6}$

\section{Die Konstruktion der Küste als Gedächtnisort tRanskUltureller Begegnungen}

In den erzählten Lebensgeschichten fast aller Figuren, ganz gleich, welcher Epoche sie angehören, geht es um Verlusterfahrungen: den Verlust der Heimat, der Freunde, der Geliebten, des Besitzes oder des Lebens. Schon in Sebalds Erzählband Die Ausgewanderten war dieses Motiv zentral, denn hier erforschte der Autor die durch erzwungenen Verlust der Heimat hervorgerufene Infragestellung kultureller Identitäten jüdischer Protagonisten und inszenierte Hotels, Sanatorien und Friedhöfe als Gedächtnisorte, in denen die traumatischen Auswirkungen dieser Unsicherheit sichtbar wurden. In seinem letzten Roman Austerlitz, der die verschütteten Wurzeln eines in London lebenden jüdischen Wissenschaftlers freilegt, übernehmen diese Funktion Bahnstationen und Wartesäle in Brüssel, London, Paris und Prag. Sie erscheinen den Protagonisten als ein »bedrohlich und unheimlich anmutende[r] Zwischenraum«, dessen »Halbdunkel« als apokalyptisches Vorzeichen einer Verfinsterung der Welt und des »Ende[s] aller Zeiten« (Pakendorf 2016: 31 u. 33) gedeutet wurde.

Die Küstenorte in den Ringen des Saturn fungieren zwar ebenfalls als Gedächtnisorte, doch sind sie weniger bedrohlich besetzt. Sie bringen vielmehr die Übergänge, Verflechtungen und Überlagerungen kultureller Identitäten zur Ansicht. Hier laufen die Fäden der erzählten Biographien von Menschen zusammen, die sich aus unterschiedlichen Gründen mit ihrer Herkunft und Identität

Gebet unterweisen sie dagegen im Eingedenken. Dieses entzauberte innen die Zukunft, der die verfallen sind, die sich bei den Wahrsagern Auskunft holen." (Benjamin 1991: 704)

6 | Was in Sebalds Text weitgehend ausgeblendet bleibt, sind jedoch die kulturellen Grenzgängerinnen. Die Vorliebe des Erzählers gilt vornehmlich männlichen Einsiedlern, Forschern, Abenteurern, Künstlern und Schriftstellern. 
auseinandersetzen müssen. Einige wurden aus ihrer Heimat vertrieben, andere suchten im nomadischen Leben zwischen den Kulturen ihre Lebensbestimmung, wieder andere reisten als Diplomaten um die Welt oder erprobten schreibend die Annäherung an fremde Kulturen.

Das Schicksal der aus der Heimat Vertriebenen wird beispielhaft an der Lebensgeschichte des jüdischen Lyrikers und Essayisten Michael Hamburger verdeutlicht, der als Kind von Berlin nach London emigrieren musste und sich später in der Nähe der ostenglischen Küste in Middleton niederließ. Die Verlusterfahrung und die Überlagerung der vertrauten Identität durch die »Stück für Stück neu erworbene Identität« (Sebald 2015: 210) ist so tiefgehend, dass kaum mehr Erinnerungsspuren zurückbleiben: »Zu viele Bauwerke sind eingestürzt, zuviel Schutt ist aufgehäuft, unüberwindlich sind die Ablagerungen und Moränen « (ebd.: 211), zitiert der Ich-Erzähler aus den Aufzeichnungen seines Freundes, den er auf seiner Wanderung in dessen Haus besucht. Allein in Träumen und Halluzinationen komme es zuweilen vor, dass die verblichenen Erinnerungen wieder Gestalt annehmen. Hamburger berichtet, dass hier die verschwundenen Orte der Kindheit in verfremdeter Form auftauchen, vermischt mit Sentenzen aus der Küstenlandschaft: Die Träume »spielen häufig in einer Umgebung, deren Merkmale teilweise auf die Weltstadt Berlin, teilweise auf das ländliche Suffolk verweisen« (ebd.: 214); »in der Traumzeit« begreife er oft nur allmählich, dass er sich nicht im Haus in Middleton, sondern in der Wohnung der Eltern in der Bleibtreustraße befinde, wo er seine Berliner Verwandtschaft, die deutschen und englischen Freunde, seine Kinder, »die Lebendigen und die Toten« (ebd. 215) versammelt finde.

Während Hamburger die Träume aufsuchen muss, um den kulturellen Verflechtungen auf den Grund zu gehen, sieht der französische Schriftsteller und Diplomat François-René Vicomte de Chateaubriand im Schreiben seiner Memoiren ein geeignetes Medium der Bewältigung von Verlusterfahrungen. Aus diesen zitierend, berichtet der Ich-Erzähler, wie jener als junger Mann vor den Schrecken der Französischen Revolution flieht, in deren Wirren zwei seiner Schwestern umkommen. In Suffolk findet der mittellose Adlige eine zweite Heimat, die er jedoch aufgrund privater Verstrickungen bald wieder verlassen muss. Sein späteres Leben, so lässt sich den Memoiren entnehmen, ist das eines ewig Reisenden, zu dessen Stationen neben London, Rom, Lausanne, Berlin und Prag auch New York, Konstantinopel und Jerusalem zählten.

Sowohl Hamburgers als auch Chateaubriands Aufzeichnungen dienen dem Erzähler als Referenzrahmen, weil hier einerseits die eigene Lebensgeschichte des zwischen den Kulturen Wandernden gespiegelt wird und andererseits Verlusterfahrungen durch den Akt des erinnernden Schreibens artikuliert und verarbeitet werden. Durch Chateaubriands Memoiren lässt der Erzähler sein eigenes poetisches Programm formulieren:

Wahr ist allerdings auch, daß ich mich meiner Erinnerungen, die so oft und so unversehens mich überwältigen, anders nicht als durch das Schreiben zu erwehren vermag. Blieben sie verschlossen in meinem Gedächtnis, sie würden schwerer und schwerer 
wiegen im Laufe der Zeit, so daß ich wohl zuletzt zusammenbrechen müßte unter ihrer ständig zunehmenden Last (ebd.: 302).

Für den vielgereisten Chateaubriand wie für den reisenden Ich-Erzähler, der im Schreiben das Grauen der Zerstörung bewältigt, scheint die literarische Arbeit »die einzig mögliche Form des Umgangs mit dem Schrecken« (Öhlschläger 2006: 313) zu sein. In ihr blitzt vorübergehend die Möglichkeit einer Erlösung auf, die im Untertitel Eine englische Wallfahrt angedeutet ist. Auch für den in Vergessenheit geratenen Spätromantiker Edward FitzGerald, dessen Grab der Erzähler in Bredfield besucht, stellt das Schreiben eine Art Erlösung dar. Einer durch Strafmaßnahmen und »maßlose Langeweile« überschatteten Kindheit entflieht der angehende Dichter, indem er die vor der »Küste kreuzenden Schiffe« (Sebald 2015: 236) beobachtet. Später wird er sich »mit dem Zusammentragen von Worten und Phrasen für ein komplettes Glossarium der Sprache der Seefahrt und des Seelebens « (ebd.: 237) beschäftigen und in dem persischen Dichter Omar Khayyam »über eine Entfernung von achthundert Jahren hinweg, seinen engsten Wahlverwandten« (ebd.: 238) entdecken. In »endlosen Stunden«, so lässt uns der Erzähler fasziniert wissen, überträgt FitzGerald das Gedicht Rubáiyát in englische Verse, die »in ihrer absichtslosen Schönheit« auf einen Punkt verweisen, »an dem das mittelalterliche Morgenland und das erlöschende Abendland einander anders als im unseligen Verlauf der Geschichte begegnen dürfen« (ebd.).

Transkulturelle Begegnungen, so die Botschaft des biographischen Erzählens, bedürfen der literarischen Erinnerungs- und Übersetzungsarbeit. Im Falle FitzGeralds sind sie auf das Übertragen der Sprache bezogen. Im Falle Hamburgers und Chateaubriands zeigen sie sich im schmerzhaften Prozess der Niederschrift von Erinnerungen, die eine Art Erlösung bieten können. Als Lebensstation der historischen Persönlichkeiten bündelt der Küstenraum die individuellen Erinnerungen, setzt sie ins Verhältnis zueinander und verdichtet sie, wodurch ein transkultureller Gedächtnisraum entsteht. Dieser Prozess soll abschließend beispielhaft am fünften Kapitel aufgezeigt werden.

Im Zentrum steht hier die Lebensgeschichte des polnisch-britischen Schriftstellers Joseph Conrad (1857-1924), dessen Entwicklung vom Waisenkind zum Seefahrer und Schriftsteller nachvollzogen wird. Das Meer avanciert in dieser Mikroerzählung zum Initiationsort, Knotenpunkt und Reflexionsraum der Lebensentscheidungen des als Konrad Korzeniowski Geborenen. Sein Vater hatte in der russischen Verbannung bezeichnenderweise Victor Hugos Roman Les travailleurs de la mer ins Polnische übersetzt. Bereits als Kind wird Conrad mit dem Verlust der Eltern konfrontiert und beschließt früh Seemann zu werden. Mit achtzehn Jahren überquert er das erste Mal den Atlantischen Ozean, drei Jahre später verschlägt es ihn als Matrose auf einen Frachter, mit dem er zahlreiche Fahrten zwischen Lowestoft und Newcastle absolviert. Der Erzähler imaginiert Conrads Aufenthalt in der englischen Küstenstadt anfänglich als den eines »Fremden « unter »Engländern«, dem dann aber »draußen auf dem Pier [...] die ihm bisher vollkommen unvertraute englische Sprache, in der er später sei- 
ne Weltruhm erlangenden Romane schreiben wird «, zugeflogen sei (ebd.: 138). Seine Weltkenntnis soll er zunächst in Form gemischter Nachrichten aus dem Lowestoft Standard bezogen haben, bevor er Jahre später selbst nach Afrika aufbrechen und als Kapitän eines Dampfbootes den Kongo befahren wird. Schon im Verlauf der langen Seereise zu seinem Bestimmungsort, so betont der Ich-Erzähler, »erkennt Korzeniowski [...] den Wahnwitz des ganzen kolonialen Unternehmens« (ebd.: 142). Nach einer mehrmonatigen beschwerlichen Reise durch das Land, die in ihm aufgrund der beobachteten Grausamkeiten an den Einheimischen »Verzweiflungsanfälle« (ebd.: 147) auslöst, welche er in seinem Roman Heart of Darkness (1899) verarbeiten wird, tritt er im Hafen von Boma die Heimreise über das Meer an.

In der historischen Person Conrads verdichten sich paradigmatisch die Verflechtungen unterschiedlicher Kulturen. Er ist Sohn polnischer Eltern, der Jahre seiner Kindheit in russischer Verbannung verbringt, als Seemann ferne Weltteile und den Kongo bereist und schließlich im Alter von dreißig Jahren die britische Staatsbürgerschaft erhält. Der Ich-Erzähler bindet diese transkulturelle Spezifik des Werdegangs eng an die Erfahrungen des Meeres. Es erweist sich als Sehnsuchts- und Fluchtort des Jugendlichen, als Reflexionsraum des in die Fremde geworfenen Seemannes und als Schreibanlass des Schriftstellers Conrad, der den kolonisierenden Zugriff der europäischen auf die afrikanische Kultur kritisch reflektiert. Korrespondierend dazu werden die Küsten- und Hafenstädte als Orte der kulturellen Begegnung und der Vernetzung mit anderen Lebensgeschichten inszeniert. So ist es kein Zufall, dass der Ich-Erzähler Conrads Rückkehr aus dem Kongo mit den Lebensstationen Joseph Loewys im Hafen von Ostende zusammenführt, auch wenn sich die historischen Personen vermutlich nie getroffen haben. ${ }^{7}$ Wir erfahren, dass Loewy, der ein Onkel des damals siebenjährigen Franz Kafka war, nach Mitwirkung am Bau des Panamakanals zwölf Jahre lang verschiedene Stellungen im kongolesischen Matadi bekleidete. In seiner Position als »Chef des gesamten Handelsdienstes « (ebd.: 148) wird er später von König Leopold persönlich ausgezeichnet, als die fertiggestellte Kongobahn in Betrieb genommen wird. Mit Conrad und Loewy lässt der Erzähler zwei Figuren aufeinandertreffen, die die Begegnung der europäischen und afrikanischen Kultur aus entgegengesetzter Perspektive erleben. Vor dem Hintergrund des erfolgsorientierten und dabei skrupellosen Unternehmers kann er Conrads kolonialkritische Haltung herausarbeiten. Während Loewy von Ostende aus in den Kongo fährt, um die »für seinesgleichen « erträglicher werdenden Lebensbedingungen zu genießen, reist Conrad in die belgische Hauptstadt, die ihm »mit ihren immer bombastischer werdenden Gebäuden wie ein [...] Grabmal« erscheint und deren Passanten ihm so vorkommen, »als trügen sie allesamt das dunkle kongolesische Geheimnis in sich« (ebd.: 148f.).

7 | In der einschlägigen Forschung zu Bezügen zwischen Conrad und Kafka wird meist nur auf diese Stelle bei Sebald verwiesen; ein Beweis für das Zusammentreffen liegt nicht vor (vgl. Lorenz 2017: 164). 
Überblendet und intensiviert wird die Wahrnehmung Conrads durch eine Erinnerung des Ich-Erzählers, der noch siebzig Jahre später eine sich in Belgien manifestierende »Verkrüppelung der Bevölkerung « erkennen will, die auf die »Zeit der ungehemmten Ausbeutung der Kongokolonie« (ebd.: 149) zurückweise. Eine weitere Überblendung geschieht durch die Einführung der Person des britischen Diplomaten Roger Casement, dessen unbeugsame Aufdeckung und Anprangerung der katastrophalen Zustände in den Kolonialgebieten sowohl Afrikas als auch Lateinamerikas eine Geistesverwandtschaft zwischen Conrad und Casement nahelegt. Beide werden als Grenzgänger inszeniert, die aufgrund ihrer transkulturellen Erfahrungen prekäre Machtverhältnisse sichtbar machen können. Während jedoch Conrad als erfolgreicher Schriftsteller agiert, bezahlt Casement sein politisches Engagement mit dem Tod. Als späterer Anhänger der irischen Befreiungsbewegung wird er nach gescheitertem Versuch, in Deutschland Waffen für die Befreiungsarmee zu rekrutieren, in London zum Tode verurteilt. Um »Gnadengesuche von einflußreicher Seite zu unterbinden«, werden aufgefundene Aufzeichnungen über seine homosexuellen Beziehungen »an den englischen König, an den Präsidenten der Vereinigten Staaten und an den Papst weitergeleitet« (ebd.: 159). Möglicherweise, so räsoniert der Ich-Erzähler, war es gerade die Homosexualität Casements, die ihn befähigte, »über die Grenzen der gesellschaftlichen Klassen und der Rassen hinweg die andauernde Unterdrückung, Ausbeutung, Versklavung und Verschrottung derjenigen zu erkennen, die am weitesten entfernt waren von den Zentren der Macht« (ebd.: 162).

Durch die Zusammenführung der unterschiedlichen Biographien wird ein Erinnerungsraum geschaffen, der vielfältige Bezüge zwischen den Kulturen sichtbar macht sowie deren kontroverse Wahrnehmung und destruktiven Aneignungsformen thematisiert. Dass dabei vom Ich-Erzähler Zusammenhänge hergestellt werden, die durch die historischen Quellen nicht immer gedeckt werden, ist von der Forschung kritisch angemerkt worden (vgl. Fuchs 2004: 199). Dem ist allerdings entgegenzusetzen, dass es Sebald weniger um eine quellenkritische Aufbereitung von Geschichte geht, als vielmehr um die Etablierung einer alternativen Erinnerungskultur, an der reale und symbolische Orte, historische Ereignisse und Assoziationen, Anekdoten und Traumsequenzen gleichermaßen teilhaben. Der Text selbst stellt dieses Potenzial zur Generierung alternativer Welten aus, wenn er sich auf Jorge Louis Borges' Erzählung Tlön, Uqbar, Orbis Tertius bezieht. Wie kaum ein anderer literarischer Text weist Borges' Erzählung auf die Problematik einer festen Grenzziehung zwischen vermeintlich wirklicher und imaginärer Welt hin, indem er die Auslöschung der bisherigen Wirklichkeit durch Objekte einer imaginierten Tlön'schen Welt beschreibt. Sebalds Erzähler deutet sie entsprechend als eine »Schrift, die in der Hauptsache befaßt ist mit unseren Versuchen zur Erfindung von Welten zweiten oder gar dritten Grades« (Sebald 2015: 89): »Eine verstreute Dynastie von Einsiedlern, die Dynastie der Erfinder, Enzyklopädisten und Lexikographen von Tlön hat das Antlitz der Erde verwandelt. Alle Sprachen, selbst Spanisch, Französisch und Englisch, werden vom Planeten verschwinden. Die Welt wird Tlön sein.« (Ebd.: 91) 
Dass Sebald seinen Erzähler fast wörtlich Borges zitieren lässt, kann als Hinweis auf das eigene poetologische Projekt genommen werden, welches mit Strukturen der Verdopplung und Vervielfältigung spielt und die Leser*innen in ein dichtes Netz von Zeichen strickt, die sie aufgrund ihrer Offenheit und Widersprüchlichkeit immer wieder neu deuten, hinterfragen und aktualisieren können. Und ähnlich, wie der imaginäre Planet Tlön einen Imaginationsraum präsentiert, in dem »nicht nur Vorstellbarkeit, sondern auch Vorstellbarmachung vorgestellt wie vorstellbar wird « (Ferretti 2015: 483), so kann die Küste als ein kultureller Erinnerungsraum gelesen werden, in dem die Konstruktion von erinnerter und imaginärer Geschichte stattfindet und zugleich vorgeführt wird.

\section{FAZIT}

Aus dem Küstenraum erzählen, heißt bei Sebald, multiperspektivisch von Begegnungen mit Kulturen zu erzählen. Die Küste ist hier nicht nur Erinnerungsanlass, sondern auch ein literarischer Ort, in dem sich disparate Gedanken, Perspektiven und Diskurse bündeln. Dabei kommen der literarischen Küste vielfältige Funktionen zu: Zunächst einmal strukturiert sie die Bewegung des Erzählers, lenkt seine Gedanken und Assoziationen, stellt Zusammenhänge zwischen seinen Erinnerungen und den erzählten Biographien her und ermöglicht Perspektiv- und Zeitenwechsel. Als konkreter Schauplatz erzeugt sie Gefühle und Gedanken, die ihren physischen Eigenschaften korrespondieren: Ihre Spezifik der permanenten Veränderung, Auflösung und Neubildung von Grenzlinien spiegelt sich in dem steten Wechsel der Bilder und Assoziationen, dem Verschwinden und unerwarteten Wiederauftauchen der Erinnerungen des Erzählers. Als Erlebnisort kann sie melancholische Stimmungen auslösen, als Projektionsraum gibt sie bestimmten kulturellen Vorstellungen und Deutungen Ausdruck. Konstruiert als katastrophisches Mnemotop, werden ihre Architekturen und Naturprozesse zu Wahrzeichen einer destruktiven Zivilisationsgeschichte. Zugleich sammeln sich hier kulturelle Energien der Vergangenheit, Gegenwart und Zukunft. Denn die Küste veranschaulicht nicht nur Zerstörung und Untergang, sondern manifestiert auch kulturelle Ordnungen, die durch die Auflösung starrer Binäroppositionen zugunsten hybrider Konstellationen gekennzeichnet sind. Die Küstenerzählung regt dazu an, Kultur in alternativen Modellen zu denken, indem sie imaginäre Überblendungen ehedem getrennter kultureller Räume erprobt. Kulturelle und sprachliche Grenzen werden durch Übersetzungsvorgänge und Perspektivwechsel verwischt, Wahrnehmungen von Kulturen kontrastiert und vervielfältigt, kulturelle Erlebnisweisen überblendet. Als liminale Zone mit unscharfen Rändern, in der sich Land und Meer beständig durchmischen, verkörpert die Küste ein Konzept, das für die Aufweichung starrer Konzepte und für Transkulturalität steht.

In Sebalds Text fungiert der Küstenraum nicht einfach als gegebener Rahmen oder bloßer Container, in dem sich Geschichten ereignen, sondern als ein sich durch die Bewegung des Ich-Erzählers konstituierendes Gefüge, das perma- 
nenten Verschiebungen und Transformationen unterworfen ist. Diese Spezifik des Transitraums Küste bildet den Ausgangspunkt und die Bedingung für die Überlagerung kultureller Erinnerungen und Begegnungen sowie für die Aushandlung der multiperspektivischen Wahrnehmung von kulturellen und literarischen Prozessen. Hier werden nicht nur die Möglichkeiten und Grenzen des Imaginierens von Welten reflektiert, sondern auch literarische Verfahren der Wiederholung und Variation, des Medien- und Perspektivwechsels, der zeitlichen Überblendung und räumlichen Verschachtelung eingesetzt, um alternative kulturelle Erinnerungsräume zu erzeugen. Die Küste, die aufgrund ihrer Grenzfiguration immer schon einen prädestinierten Raum kultureller Aushandlungen darstellte, in dem sich Materialien, Ideen und Visionen überlappen und durchdringen, avanciert in den Ringen des Saturn zu einem Ort, an dem Prozesse der Verflüssigung und Verdichtung, der Überblendung und Vervielfältigung kultureller Wahrnehmung erzählerisch ausbuchstabiert werden.

\section{LiterATUR}

Albes, Claudia (2002): Die Erkundung der Leere. Anmerkungen zu W.G. Sebalds "englischer Wallfahrt" Die Ringe des Saturn. In: Jahrbuch der Deutschen Schillergesellschaft 46, S. 279-305.

Bader, Hannah (2008): Gischt. Zu einer Geschichte des Meeres. In: Dies. / Gerhard Wolf (Hg.): Das Meer, der Tausch und die Grenzen der Repräsentation. Zürich, S. 15-40.

Benjamin, Walter (1991): Über den Begriff der Geschichte. In: Ders.: Gesammelte Schriften. Bd. I/2. Hg. v. Rolf Tiedemann u. Hermann Schweppenhäuser. Frankfurt a.M., S. 691-704.

Bonner, Withold/Egger, Sabine/Hess-Lüttich, Ernest W.B. (2016): Vom Bahnhof zum Raum der Übersetzung. Transiträume in Literatur, Kultur und Sprache. In: Zeitschrift für interkulturelle Germanistik 7, H. 2, S. 11-19.

Corbin, Alain (21999): Meereslust. Das Abendland und die Entdeckung der Küste. Aus dem Franz. v. Grete Osterwald. Frankfurt a.M.

Feldbusch, Thorsten (2003): Zwischen Land und Meer. Schreiben auf den Grenzen. Würzburg.

Ferretti, Victor A. (2015): [Art.] "Tlön: Imaginationsräume und Ander-Welten«. In: Jörg Dünne/Andreas Mahler (Hg.): Handbuch Literatur \& Raum. Berlin/Boston, S. 478484.

Fuchs, Anne (2004): Die Schmerzensspuren der Geschichte. Zur Poetik der Erinnerung in W.G. Sebalds Prosa. Köln.

Kastura, Thomas (1996): Geheimnisvolle Fähigkeit zur Transmigration. W.G. Sebalds interkulturelle Wallfahrten in die Leere. In: Arcadia 31, H. 1/2, S. 197-216.

Kelletat, Dieter (1999): Physische Geographie der Meere und Küsten. Eine Einführung. Stuttgart/Leipzig.

Kimmich, Dorothee/Schahadat, Schamma (2012): Einleitung. In: Dies. (Hg.): Kulturen in Bewegung. Beiträge zur Theorie und Praxis der Transkulturalität. Bielefeld, S. 7-21. 
Lemke, Anja (2008): Figurationen der Melancholie. In: Zeitschrift für deutsche Philologie 127, H. 2, S. 97-125.

Lorenz, Matthias N. (2017): Distant Kinship - Entfernte Verwandtschaft: Joseph Conrads "Heart of Darkness" in der deutschen Literatur von Kafka bis Kracht. Stuttgart.

Mosbach, Bettina (2008): Figurationen der Katastrophe. Ästhetische Verfahren in W.G. Sebalds Die Ringe des Saturn und Austerlitz. Bielefeld.

Öhlschläger, Claudia (2006): Die Ringe des Saturn: ein kosmologisches Strukturmodell für W.G. Sebalds Lektüre zivilisatorischer Abirrungen. In: Maximilian Bergengruen/Davide Giuriato/Sandro Zanetti (Hg.): Gestirne und Literatur im 20. Jahrhundert. Frankfurt a.M., S. 312-325.

Dies. (2017): [Art.] "Die Ringe des Saturn". In: Dies./Michael Niehaus (Hg.): SebaldHandbuch. Leben - Werk - Wirkung. Stuttgart 2017, S. 38-47.

Pakendorf, Gunther (2016): Austerlitz im Wartesaal. In: Zeitschrift für interkulturelle Germanistik 7, H. 2, S. 21-34.

Pethes, Nicolas (2015): [Art.] "Mnemotop«. In: Jörg Dünne/Andreas Mahler (Hg.): Handbuch Literatur \& Raum. Berlin/Boston, S. 196-204.

Ratzel, Friedrich (1911): Das Meer als Quelle der Völkergröße. Eine politisch-geographische Studie. München/Berlin.

Sebald W.G. (1985): Helle Bilder und dunkle. Zur Dialektik der Eschatologie bei Stifter und Handke. In: Ders.: Die Beschreibung des Unglücks. Zur österreichischen Literatur von Stifter bis Handke. Salzburg/Wien, S. 165-186.

Ders. (2015): Die Ringe des Saturn. Eine englische Wallfahrt. Frankfurt a.M.

Wirth, Uwe (2012): Zwischenräumliche Bewegungspraktiken. In: Ders. (Hg.): Bewegen im Zwischenraum. Berlin, S. 7-34.

Wolf, Burckhardt (2013): Fortuna di mare. Literatur und Seefahrt. Zürich/Berlin. 